\title{
Status of Borexino experiment and Physics results with its Counting Test Facility (CTF)
}

\author{
Barbara Caccianiga ${ }^{* \dagger}$ \\ Dipartimento di Fisica dell'Università di Milano and INFN, I-20133, Italy \\ E-mail: barbara.caccianiga@mi.infn.it
}

\begin{abstract}
Borexino is a solar neutrino experiment located under the Gran Sasso mountain in Italy. It is currently in the commissioning phase and will start taking data in 2007. Its main goal is to detect low-energy neutrinos coming from the sun in real-time, via the scattering reaction $v e^{-} \rightarrow v e^{-}$. Borexino exploits 300 tons of liquid scintillator as active target for neutrinos and 2200 photomultiplier tubes mounted on a stainless steel sphere $(\mathrm{d}=14 \mathrm{~m})$ to view the scintillation light emitted by the recoil electron. In order to lower the experimental threshold down to $250 \mathrm{keV}$, it is necessary to keep unprecedented levels of radioupurity of the scintillator and of all the materials surrounding it. A prototype of Borexino, the Counting Test Facility (CTF) has been built and operated since 1994 to prove the capability to reach such levels of radiopurity. Besides its main goal of pilot experiment for Borexino, the high mass and high radiopurity of CTF have made it a unique detector to explore rare or forbidden processes with unprecedented sensitivity. Some of the physics results obtained in the last years with CTF are presented: they include limits on neutrino magnetic moment, electron lifetime, Pauli Exclusion Principle violation, nucleon decay and anti-neutrino flux from the sun.
\end{abstract}

International Europhysics Conference on High Energy Physics

July 21 st - 27th 2005

Lisboa, Portugal

\footnotetext{
* Speaker.

$\dagger$ On behalf of the Borexino collaboration.
} 

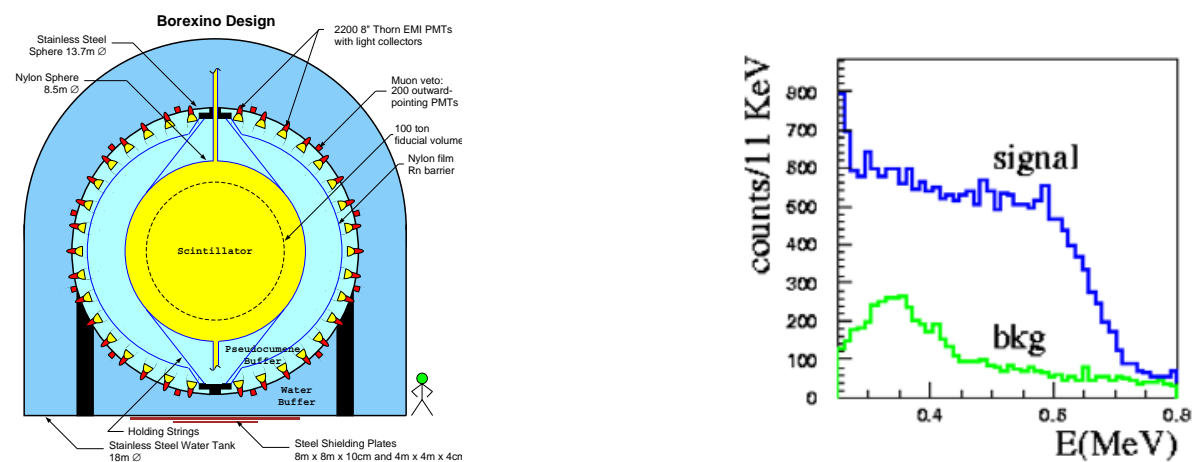

Figure 1: A schematic view of the Borexino detector (left); the signal and background shape expected in Borexino according to MonteCarlo simulation (right)

\section{Introduction}

The long standing solar neutrino problem has been explained with the phenomenon of neutrino oscillations. In this scenario, the combined fit to the results of all solar neutrino experiments plus the reactor experiment Kamland has restricted the allowed region in the parameter space $\left(\Delta m^{2}, \tan ^{2} \theta\right)$ to the so-called Large Mixing Angle (LMA) solution. The best fit values are $\Delta m^{2}=7.9_{-0.5}^{+0.6} \times 10^{-5} \mathrm{eV}^{2}$ and $\tan ^{2} \theta=0.40_{-0.07}^{+0.10}$. So far, the solar neutrino oscillation hypothesis and the Standard Solar Model have been tested in real-time only on high energy neutrinos $(\mathrm{E}>5 \mathrm{MeV})$ which represent a small portion $(<1 \%)$ of the solar neutrino flux; a direct detection of the low-energy part of the solar spectrum is therefore needed. In particular, it is important to measure the ${ }^{7} \mathrm{Be}$ neutrino flux which is currently known with the highest experimental uncertainty. Furthermore, detecting neutrinos around $1 \mathrm{MeV}$ will probe oscillations in an energy region where the transition between vacuum-dominated and matter-dominated effect occurs, making it possible to evidentiate non-standard effects (see reference [1]). Borexino will be the first real-time detector to adress these important issues.

\section{Borexino}

Borexino is depicted schematically in figure 1 . The detector core consists of 300 tons of liquid scintillator (pseudocumene $+1.5 \mathrm{~g} / \mathrm{g}$ of PPO) contained in a spherical vessel (diameter $=8.5 \mathrm{~m}$ ) and immersed in 1000 tons of buffer liquid which provides shielding against external radioactivity. The light emitted by the scintillator is detected by 2200 photomultiplier tubes mounted on a stainless steel sphere (diameter $=14 \mathrm{~m}$ ) concentrical with the inner vessel. The apparatus is surrounded by 2000 tons of water in a cylindrical dome, approximately $20 \mathrm{~m}$ high and $15 \mathrm{~m}$ large [2].

The main goal of Borexino is the detection of neutrinos from the sun via the scattering process $e^{-} v_{x} \rightarrow e^{-} v_{x}$. The neutrino signature is given by the scintillation light produced as the recoil electron deposits its energy in the medium. Unlike the other projects which exploit the same detecting reaction, Borexino is planned to work with a very low energy threshold $(250 \mathrm{keV})$ which corresponds to a minimum detectable neutrino energy of approximately $400 \mathrm{keV}$, thus providing important information on the low energy portion of the solar neutrino spectrum. The expected rate 

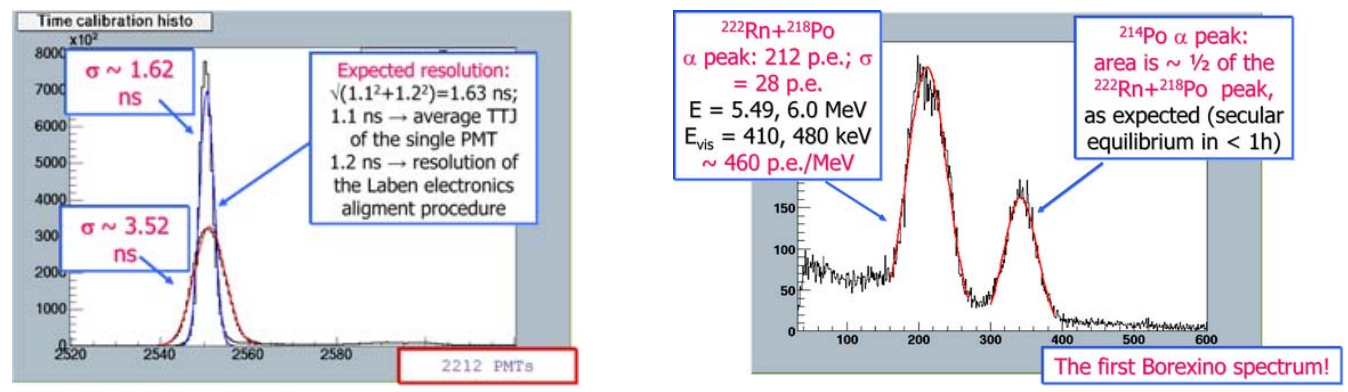

Figure 2: Air-run of Borexino: time response of the 2200 PMTs to a synchronous laser pulse before and after alignment (left); energy spectrum (number of collected photon electrons) of the Rn source (right)

of ${ }^{7} \mathrm{Be}$ and $p p$ neutrinos in Borexino is of $\sim 35$ ev/day and $\sim 1$ ev/day respectively. For the experiment to be able of disantangling this signal, it is mandatory to keep background from natural radioactivity and from external sources at very low levels [3]. The external background contribution is reduced to acceptable levels thanks to the shielding strategy based on concentrical shells of increasingly pure materials. The internal background issue is adressed by applying sophisticated purification techniques (such as distillation and water extraction) to bring the content of radioactive contaminants in the scintillator to the required levels $\left(10^{-16} \mathrm{~g} / \mathrm{g}\right.$ in $\mathrm{U}$ and $\mathrm{Th}, 10^{-14} \mathrm{~g} / \mathrm{g}$ in $\mathrm{K})$. With this levels of radioactivity the proportion between signal and background in the so-called neutrino window would be the one shown in figure 1. The capability to reach the radiopurity levels needed for Borexino has been proven by a prototype of the experiment, the Counting Test Facility, which has been built and operated since 1994 [4] (see paragraph 4 for details on other results of CTF).

Even though its main focus will be on solar neutrino physics, Borexino has the potential to give significant contributions to other neutrino fields, such as geo-neutrinos and supernova neutrinos. In particular, the signal to background ratio for what concerns geo-neutrinos will be favourable in Borexino with respect to other experiments (such as Kamland), since Italy doesn't have reactor plants.

\section{Status of the Borexino detector}

The detector installation has been completed in july 2004. Since then, several campaigns of data-taking with the empty detector (the so-called air-runs) have been performed successfully to check the overall performance of the apparatus, DAQ, electronics and software. The laser system for the PMT time equalization has been tested in its final configuration and proved to be working within specifications, allowing to align the time response of the 2200 phototubes witin $1.6 \mathrm{nsec}$ (see figure 2). Some runs were taken inserting in the detector a radioactive source (a vial containing liquid scintillator loaded with $\mathrm{Rn}$ ) to check the light collection efficiency of the apparatus and to test the position reconstruction algorithm. Figure 2 shows the energy spectrum collected during these runs. The average spatial resolution obtained in this test was around $12 \mathrm{~cm}$. The detector will be filled with pseudocumene in 2006 and will be ready to take data by the beginning of 2007 . 


\section{Physics results with the Counting Test Facility}

CTF consists of 4 tons of liquid scintillator contained in a 1-meter-radius nylon vessel and surrounded by 100 photomultiplier tubes pointing towards the center. It is a unique detector, whose size and low contamination levels make it capable of searching for rare and forbidden processes in the low energy region $(\sim 1 \mathrm{MeV})$ with high sensitivity. The first important result obtained with CTF concerns the electron stability: the data have been analyzed to search for the $256 \mathrm{keV}$ line of the $\gamma$ emitted in the decay channel $e^{-} \rightarrow \gamma v$. No signal was found and this enabled to set a limit on the electron lifetime of $\tau>4.6 \times 10^{26}$ years (90\% C.L.) which was published in 2002 and is still the best limit in the world for the electron decay in this channel [5]. The analysis of the CTF data has also addressed the neutrino magnetic moment question: a non-zero $\mu_{4}$ would increase the cross-section of neutrino-electron elastic scattering. This effect becomes dominant at low energy ( $<400 \mathrm{keV}$ ) which makes CTF particularly sensitive to $\mu_{v}$. A limit $\mu_{v}<5 \times 10^{-10} \mu_{B}$ was obtained which is still the best result with low-energy neutrinos[8]. The stability of nucleons bounded in nuclei has been studied in CTF searching for the decays of single nucleon or pair of nucleons into invisible channels: if one of such decays occurs inside ${ }^{12} \mathrm{C},{ }^{13} \mathrm{C},{ }^{16} \mathrm{O}$, daughter nuclei would be produced such as ${ }^{11} \mathrm{C},{ }^{10} \mathrm{C},{ }^{12} \mathrm{~B},{ }^{11} \mathrm{Be},{ }^{14} \mathrm{O}$ which in turn would decay radioactively. The limits obtained with CTF are comparable to or improve the previously set world limits [6]. In 2004 an article was published concerning the search for non-Paulian transitions of nucleons from $1 \mathrm{P}$ shell to a filled $1 \mathrm{~S}_{1 / 2}$ shell to put limits on the Pauli Esclusion Principle. The analysis consists in searching for $\alpha$, p, n, $\gamma$ emitted in non-Paulian decays of ${ }^{12} C$ e ${ }^{16} O$ nuclei. The obtained limits significantly improves (up to 3 orders of magnitude) the previously set limits [9]. Other studies performed on the CTF data concern the production of heavy-neutrinos in the sun [7] and the flux of anti-neutrinos from the sun (this last work is still in progress and will be published shortly).

\section{Conclusions}

Borexino has been built and will start taking data in 2007. Its prototype CTF has demostrated the capability to reach the high radiopurity levels needed. Besides this, it allowed searches for rare and forbidden processes with high sensitivity.

\section{References}

[1] Miranda et al, [hep-ph/0406280]; Vissani et al, [hep-ph/0403158].

[2] G. Alimonti et al, Astr. Phys. 16 (2002) 205.

[3] C. Arpesella et al., Astr. Phys. 18 (2002) 1.

[4] G. Alimonti et al., NIM A 406 (1998) 411.

[5] H.O. Back et al., Phys. Lett B525 (2002) 29.

[6] H.O. Back et al., Phys. Lett B563 (2003) 23.

[7] H.O. Back et al., Pis'ma v ZhETF 78, iss.5 (2003) 707.

[8] H.O. Back et al.,Phys. Lett B563 (2003) 35.

[9] H.O. Back et al., Eur. Phys. J. C37 (2004) 421. 